\title{
New stage in high-energy gamma-ray studies with GAMMA-400 after Fermi-LAT
}

\author{
N.P. Topchiev ${ }^{1, \text { a }}$, A.M. Galper ${ }^{1,2}$, V. Bonvicini ${ }^{3}$, O. Adriani ${ }^{4}$, I.V. Arkhangelskaja ${ }^{2}$, A.I. Arkhangelskiy ${ }^{2}$, A.V. Bakaldin ${ }^{5}$, \\ S.G. Bobkov ${ }^{5}$, M. Boezio ${ }^{3}$, O.D. Dalkarov ${ }^{1}$, A.E. Egorov ${ }^{1}$, M.S. Gorbunov ${ }^{5}$, Yu.V. Gusakov ${ }^{1}$, B.I. Hnatyk ${ }^{6}$, V.V. \\ Kadilin $^{2}$, V.A. Kaplin ${ }^{2}$, M.D. Kheymits ${ }^{2}$, V.E. Korepanov ${ }^{7}$, A.A. Leonov ${ }^{1,2}$, F. Longo ${ }^{3}$, V.V. Mikhailov ${ }^{2}$, E. Mocchiutti ${ }^{3}$, \\ A.A. Moiseev ${ }^{8}$, I.V. Moskalenko ${ }^{9}$, P.Yu. Naumov ${ }^{2}$, P. Picozza ${ }^{10}$, M.F. Runtso ${ }^{2}$, O.V. Serdin ${ }^{5}$, R. Sparvoli ${ }^{10}$, P. Spillantini ${ }^{4}$, \\ Yu.I. Stozhkov ${ }^{1}$, S.I. Suchkov ${ }^{1}$, A.A. Taraskin ${ }^{2}$, M. Tavani ${ }^{11}$, Yu.T. Yurkin ${ }^{2}$, and V.G. Zverev ${ }^{1}$ \\ 1 Lebedev Physical Institute, 119991 Moscow, Russia \\ 2 National Research Nuclear University MEPhI (Moscow Engineering Physics Institute), Kashirskoe highway 31, Moscow 115409, \\ Russia \\ 3 Istituto Nazionale di Fisica Nucleare, Sezione di Trieste, 34149 Trieste, Italy \\ ${ }^{4}$ Istituto Nazionale di Fisica Nucleare, Sezione di Florence, 50019 Sesto Fiorentino, Florence, Italy \\ 5 Scientific Research Institute for System Analysis, 117218 Moscow, Russia \\ 6 Taras Shevchenko National University, Kyiv 01601, Ukraine \\ 7 Lviv Center of Institute of Space Research, Lviv 79060, Ukraine \\ 8 NASA Goddard Space Flight Center and CRESST/University of Maryland, Greenbelt, Maryland 20771, USA \\ 9 Hansen Experimental Physics Laboratory and Kavli Institute for Particle Astrophysics and Cosmology, Stanford University, \\ Stanford, CA 94305, USA \\ 10 Istituto Nazionale di Fisica Nucleare, Sezione di Rome "Tor Vergata", 00133 Rome, Italy \\ 11 Istituto Nazionale di Astrofisica IASF and Physics Department of University of Rome Tor Vergata, 00133 Roma, Italy
}

\begin{abstract}
Fermi-LAT has made a significant contribution to the study of high-energy gamma-ray diffuse emission and the observations of 3000 discrete sources. However, one third of all gamma-ray sources (both galactic and extragalactic) are unidentified, the data on the diffuse gamma-ray emission should be clarified, and signatures of dark matter particles in the high-energy gamma-ray range are not observed up to now. GAMMA-400, the currently developing gamma-ray telescope, will have angular $\left(\sim 0.01^{\circ}\right.$ at $\left.100 \mathrm{GeV}\right)$ and energy $(\sim 1 \%$ at $100 \mathrm{GeV})$ resolutions in the energy range of $10-1000 \mathrm{GeV}$ which are better than Fermi-LAT (as well as ground gamma-ray telescopes) by a factor of 5-10. It will observe some regions of the Universe (such as the Galactic Center, Fermi Bubbles, Crab, Cygnus, etc.) in a highly elliptic orbit (without shading the telescope by the Earth) continuously for a long time. It will allow us to identify many discrete sources, to clarify the structure of extended sources, to specify the data on the diffuse emission, and to resolve gamma rays from dark matter particles.
\end{abstract}

\section{Current gamma-ray study challenges}

\subsection{Analysis of gamma-ray results according to the Fermi-LAT and ground-based telescope data}

Since 2008 Fermi-LAT is operating in a near-Earth orbit in the scanning mode and surveying full sky every three hours. Up to now, three catalogs of gamma-ray sources have been published based on the Fermi-LAT observational results: 1FGL [1] and 2FGL [2] for the energy range from $100 \mathrm{MeV}$ to $100 \mathrm{GeV}$, 3FGL [3] for the energy range from $100 \mathrm{MeV}$ to $300 \mathrm{GeV}$. Moreover two catalogs of high-energy gamma-ray sources were published: 1FHL [4] for energies above $10 \mathrm{GeV}$ and 2FHL [5] for the energy range of $50 \mathrm{GeV}-2 \mathrm{TeV}$. Figure 1 [6] shows the percentage of the different types of gamma-ray sources according to 3FGL. It is seen that $33 \%$ of gamma-ray sources are unidentified. In [3] the exposure of the source observations according to 3FGL is presented.
It is seen that during four years of operation Fermi-LAT observed the Galactic center during only $12 \%$ or $1 / 8$ of the total operation time.

Based on results of gamma-ray observations at energies above $100 \mathrm{GeV}$ by ground-based facilities VERITAS [7] MAGIC [8] H.E.S.S. [9] and others, the TeVCat catalog (http://tevcat.uchicago.edu/) of discrete gamma-ray sources was created, which contains about 180 sources and mainly matches with 3FGL.

It is important to note that the observational data from Fermi-LAT and ground-based facilities were obtained for the energy ranges which overlap poorly for many gamma-ray sources. Sometimes they don't overlap at all. Hence, the frontier range around $100 \mathrm{GeV}$ is still very interesting for investigations. In addition, the angular resolution of Fermi-LAT, existing groundbased telescopes, and even the planned CTA [10] in the region of around $10-300 \mathrm{GeV}$ is only $\sim 0.1^{\circ}$. Therefore a

a e-mail: tnp51@yandex.ru

(C) The Authors, published by EDP Sciences. This is an Open Access article distributed under the terms of the Creative Commons Attribution License 4.0 (http://creativecommons.org/licenses/by/4.0/). 


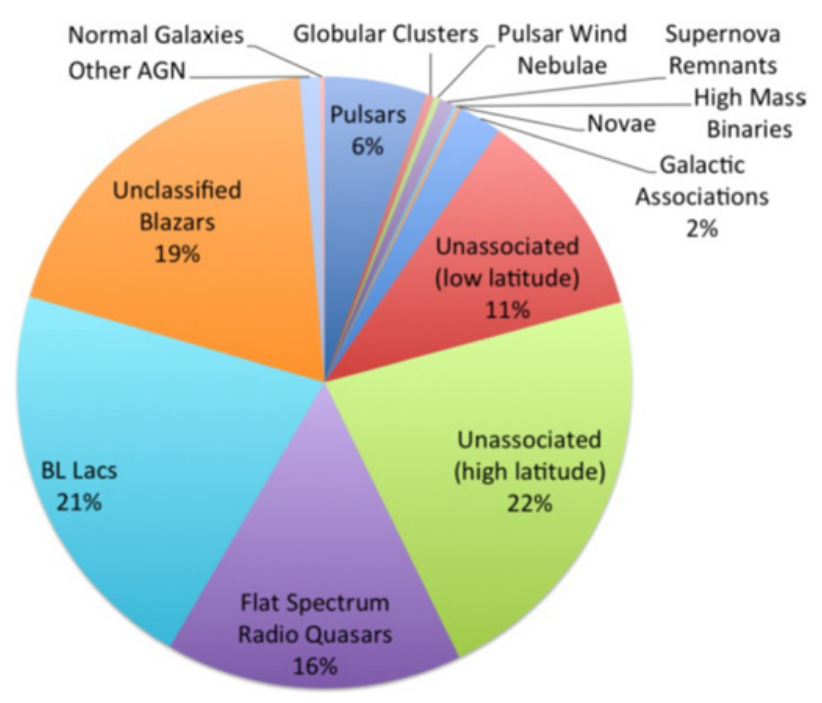

Figure 1. The percentage of the different types of gamma-ray sources [6] according to the 3FGL.
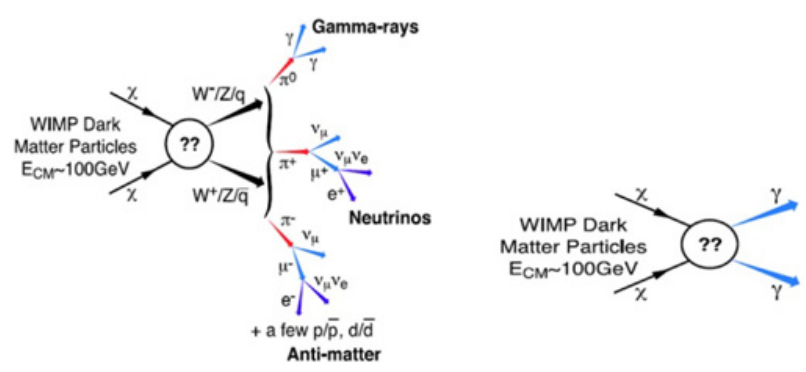

Figure 2. Possible channels of WIMP annihilation $\chi \chi \rightarrow$ $b \bar{b}, \tau^{+} \tau^{-}, W^{+} W^{-}, \mu^{+} \mu^{-}, q \bar{q}, Z Z$ with gamma-ray production [12].

much better angular resolution is required in order to identify many gamma-ray sources.

\subsection{Indirect searches of dark matter}

Another very interesting and important goal in the studies of the gamma-ray sky is indirect searches of dark matter (DM). In general, an exact physical nature of DM is a top puzzle in modern astrophysics. Many candidates for the DM role are being proposed. However, WIMPs with mass between several $\mathrm{GeV}$ and several $\mathrm{TeV}$ are still considered as the most probable candidate [11]. WIMPs can annihilate or decay with the production of gamma rays. This emission can have both a continuum energy spectrum or monoenergetic lines. This depends on which annihilation channel realizes in nature. The continuum spectrum would come in the case of annihilation into particle pairs like $\chi \chi \rightarrow b \bar{b}, \tau^{+} \tau^{-}, W^{+} W^{-}, \mu^{+} \mu^{-}, q \bar{q}, Z Z$ or others and gamma-ray lines would be produced in the case of direct annihilation into photons $\chi \chi \rightarrow \gamma \gamma, \gamma Z, \gamma H$ (Fig. 2 [12]).

To resolve gamma-ray lines from the background it is necessary to have a high-energy resolution. Figure 3 shows the expected energy spectrum for the annihilation of 300$\mathrm{GeV}$ WIMPs producing gamma rays ( $\gamma \gamma, \gamma Z, \gamma H$ lines), which can be resolved from the background by various telescopes with energy resolutions of $10 \%, 5 \%$, and

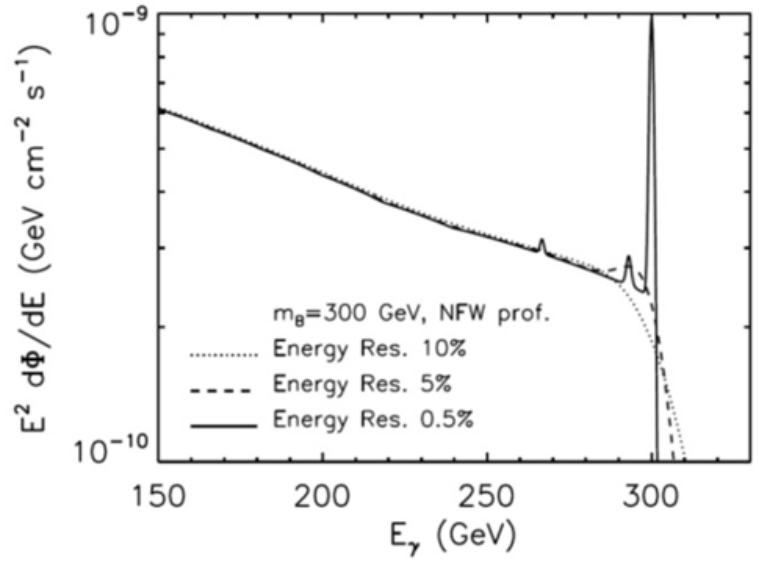

Figure 3. Expected energy spectrum for the annihilation of 300$\mathrm{GeV}$ WIMP producing gamma rays $(\gamma \gamma, \gamma Z, \gamma H$ lines), which can be resolved from the background by various telescopes with energy resolutions of $10 \%, 5 \%$, and $0,5 \%$ [13].

$0,5 \%$ [13]. Note that the energy resolution of Fermi-LAT and ground-based facilities is only $10-15 \%$ at energies of 10-300 GeV. Thus, as seen from Fig. 3, future telescopes need to have $1-2 \%$ energy resolution.

\section{THE GAMMA-400 GAMMA-RAY TELESCOPE}

Thus, to resolve unidentified gamma-ray sources and search for potential gamma-ray lines from DM we need a gamma-ray telescope with an angular resolution of several hundredth degrees and an energy resolution of a few percent for an energy of $\sim 100 \mathrm{GeV}$. This is going to be GAMMA-400, which will be installed onboard the Russian space observatory [14-18].

The GAMMA-400 main scientific goals are: a search for dark matter by means of gamma-ray astronomy; precise measurements of the Galactic Center, Fermi Bubbles, Crab, Vela, Cygnus, Geminga, Sun, and other regions, extended and point gamma-ray sources, diffuse gamma rays with unprecedented angular $\left(\sim 0.01^{\circ}\right.$ at $\left.E_{\gamma}>100 \mathrm{GeV}\right)$ and energy $\left(\sim 1 \%\right.$ at $\left.E_{\gamma}>100 \mathrm{GeV}\right)$ resolutions.

\subsection{The GAMMA-400 physical scheme}

The physical scheme of the GAMMA-400 gammaray telescope is shown in Fig. 4. The GAMMA-400 energy range for gamma-ray studies is from $\sim 20 \mathrm{MeV}$ to $\sim 1000 \mathrm{GeV}$. The GAMMA-400 field of view $(\mathrm{FoV})$ is $\pm 45^{\circ}$.

GAMMA-400 consists of plastic scintillation anticoincidence top and lateral detectors (ACtop and AClat), a converter-tracker (C), plastic scintillation detectors ( $\mathrm{S} 1$ and $\mathrm{S} 2)$ for the time-of-flight system (ToF), calorimeter (CC), plastic scintillation detector (S3).

The anticoincidence detectors surrounding the convertertracker are used to distinguish gamma rays from a significantly larger number of charged particles (e.g., in the region of $10-100 \mathrm{GeV}$, the flux ratios for gamma rays to electrons and protons are $\sim 1: 10^{2}: 10^{5}$.

All scintillation detectors consist of two independent 1-cm layers. The time-of-flight system, where detectors 


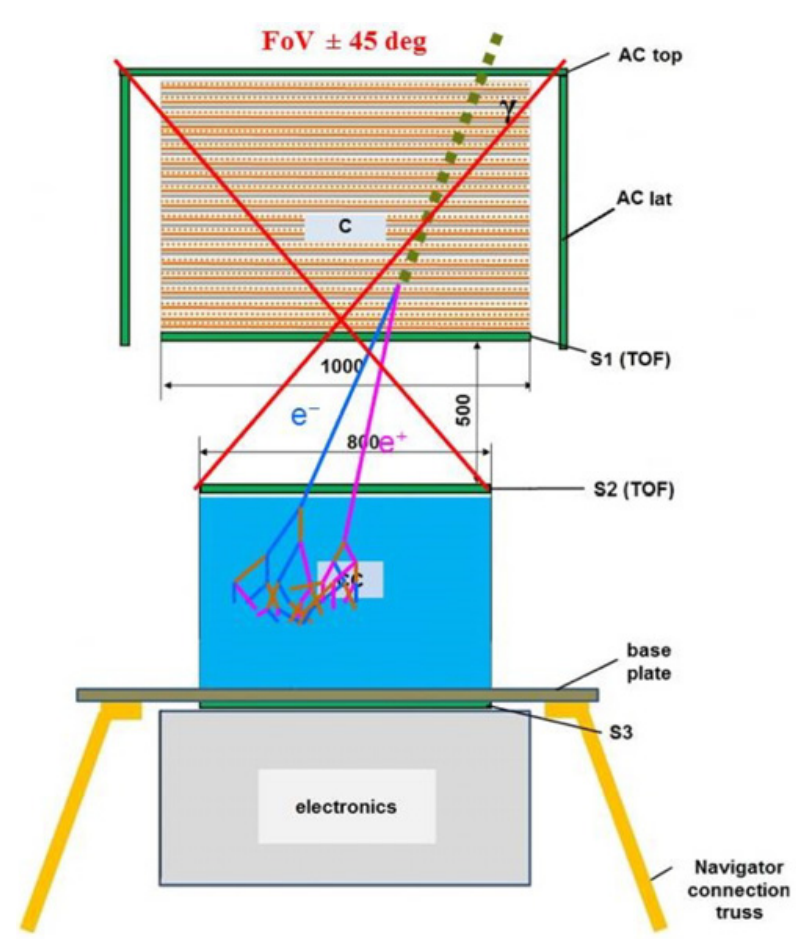

Figure 4. The GAMMA-400 physical scheme.

$\mathrm{S} 1$ and S2 are separated by approximately $500 \mathrm{~mm}$, determines the top-down direction of arriving particles. The additional scintillation detector S3 improves hadron and electromagnetic shower separation.

The converter-tracker consists of 22 layers of double (x,y) silicon strip coordinate detectors (pitch of $0.08 \mathrm{~mm}$ ). Twenty layers are interleaved with tungsten conversion foils and the final two layers have no tungsten. This configuration allows us to measure gamma rays down to approximately $20 \mathrm{MeV}$. The total converter-tracker thickness is about 1 radiation length, $X_{0}$. The convertertracker information is used to precisely determine the conversion point and the direction of each incident particle.

The calorimeter CC measures the particle energy and consists of $\mathrm{CsI}(\mathrm{Tl})$ crystals. The total calorimeter thickness is $\sim 21 X_{0}$ or $\sim 1.0 \lambda_{0}$ (where $\lambda_{0}$ is the nuclear interaction length) when detecting vertical incident particles and $\sim 43 X_{0}$ or $\sim 2.0 \lambda_{0}$ when detecting laterally incident particles. Using the deep calorimeter allows us to extend the energy range up to several $\mathrm{TeV}$ for gamma rays, and to reach an energy resolution of approximately $1 \%$ above $100 \mathrm{GeV}$.

\subsection{The GAMMA-400 gamma-ray observatory}

The GAMMA-400 gamma-ray observatory will be installed onboard the Navigator space platform, which is designed and manufactured by the Lavochkin Association.

Using the Navigator space platform gives the GAMMA-400 experiment a highly unique opportunity for the near future gamma- and cosmic-ray science, since it allows us to install a scientific payload (mass of $2500 \mathrm{~kg}$, power consumption of $2000 \mathrm{~W}$, and telemetry downlink of $100 \mathrm{~GB} /$ day, with a lifetime more than 7 years), which will provide GAMMA-400 with the means to significantly contribute as the next generation instrument for gammaray astronomy and cosmic-ray physics.
The GAMMA-400 experiment will be initially launched into a highly elliptical orbit (with an apogee of $300,000 \mathrm{~km}$ and a perigee of $500 \mathrm{~km}$, with an inclination of $51.4^{\circ}$ ), with 7 days orbital period. Under the action of gravitational disturbances of the Sun, Moon, and the Earth after $\sim 6$ months the orbit will transform to about an approximately circular one with a radius of $\sim 200000 \mathrm{~km}$ and will not suffer from the Earth's occultation and shielding by the radiation belts. A great advantage of such an orbit is the fact that the full sky coverage will always be available for gamma-ray astronomy, since the Earth will not cover a significant fraction of the sky, as is usually the case for a low-Earth orbit. Therefore, the GAMMA400 source pointing strategy will hence be properly defined to maximize the physics outcome of the experiment. The launch of the GAMMA-400 space observatory is planned for the middle of the 2020s.

\subsection{Comparison of GAMMA- 400 with Fermi-LAT and ground-based facilities}

GAMMA-400 has numerous advantages in comparison with Fermi-LAT:

- a highly elliptical orbit (without the Earth's occultation and away from the radiation belts) allows us to observe with the full aperture of $\pm 45^{\circ}$ different gamma-ray sources continuously over a long period of time with an exposure greater by a factor of 8 than for FermiLAT operating in the sky-survey mode;

- thanks to a smaller pitch (by a factor of 3) and analog readout in the coordinate silicon strip detectors, GAMMA-400 has an excellent angular resolution above $\sim 20 \mathrm{MeV}$;

- due to the deep $\left(\sim 21 X_{0}\right)$ calorimeter, GAMMA-400 has an excellent energy resolution and can detect gamma rays more reliably up to several $\mathrm{TeV}$ for vertically incident events;

- owing to the better gamma-ray separation from cosmic rays (in contrast to Fermi-LAT, the presence of a special trigger with event timing, time-of-flight system, two-layer scintillation detectors), GAMMA-400 is significantly well equipped to separate gamma rays from the background of cosmic rays and backscattering events.

GAMMA-400 will also have better angular and energy resolutions in the energy region $10-1000 \mathrm{GeV}$ in comparison with current and future space- and ground-based instruments (VERITAS [7], MAGIC [8], H.E.S.S. [9], CTA [10], and HAWC [19] (Figs. 5, 6) and will allow us to fill the gap at energies of $\sim 100 \mathrm{GeV}$ between the spaceand ground-based instruments.

\section{The anticipated GAMMA-400 scientific output}

\subsection{Galactic plane}

GAMMA-400 will study continuously over a long period of time different regions of the Galatic plane (Fig. 7), for example, the Galactic center, Fermi Bubbles, Crab, Vela, Cygnus, Geminga with FoV of $\pm 45^{\circ}$. In particular, using the gamma-ray fluxes obtained by Fermi-LAT, we can expect that GAMMA-400, when observing the Galactic 


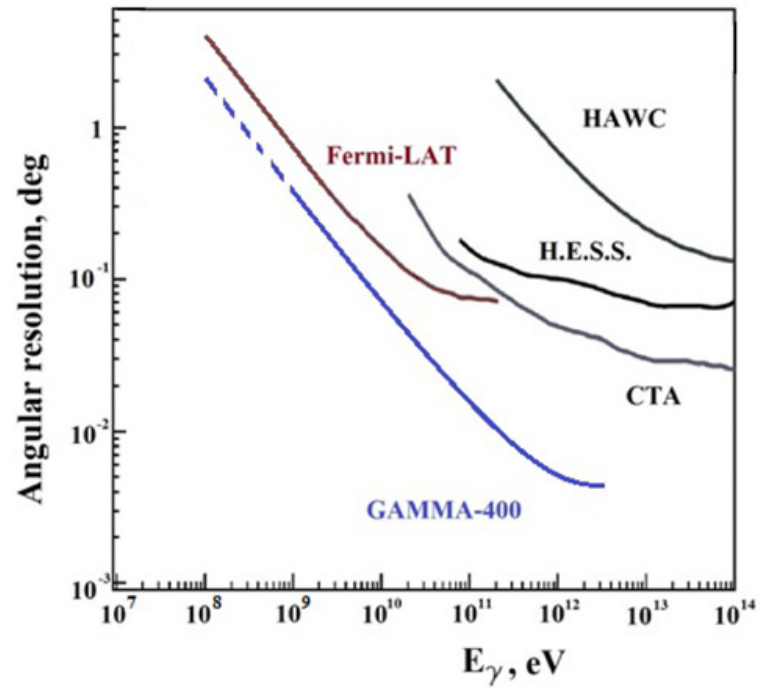

Figure 5. Comparison of angular resolutions for GAMMA-400, Fermi-LAT, H.E.S.S., HAWC, and CTA.

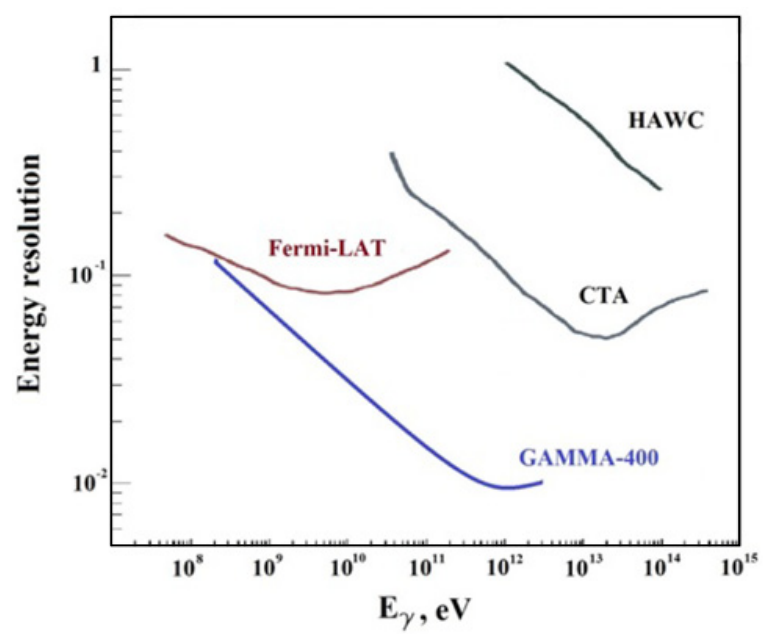

Figure 6. Comparison of energy resolutions for GAMMA-400, Fermi-LAT, H.E.S.S., HAWC, and CTA.

center with an aperture of $\pm 45^{\circ}$ during 1 year will detect: 57400 photons for $E_{\gamma}>10 \mathrm{GeV} ; 5240$ photons for $E_{\gamma}>$ $50 \mathrm{GeV} ; 1280$ photons for $E_{\gamma}>100 \mathrm{GeV} ; 535$ photons for $E_{\gamma}>200 \mathrm{GeV}$.

\subsection{Dark matter searching}

When detecting gamma rays from dark matter the intensity from gamma rays is calculated by

$$
\begin{gathered}
\frac{d \phi}{d \Omega d E}=\frac{1}{2} \frac{\langle\sigma \nu\rangle}{m_{\chi}^{2}} \frac{d N_{\gamma}}{d E} \times \frac{J}{4 \pi} \\
{\left[\mathrm{erg} \mathrm{s}^{-1} \mathrm{~cm}^{-2} \mathrm{GeV}^{-1} \mathrm{sr}^{-1}\right]} \\
J=\int_{l o s} \rho^{2}(\vec{r}) d l
\end{gathered}
$$

Here, the first term contains the information only on dark matter particle: $\langle\sigma \nu\rangle$ is the particle annihilation cross section, $m_{\chi}$ is the particle mass, $d N_{\gamma} / d E$ is the spectrum of gamma rays from annihilation (http:// www.marcocirelli.net/PPPC4DMID.html). The second term is determined using the distribution of dark matter density $\rho(r)$ in the object under study.

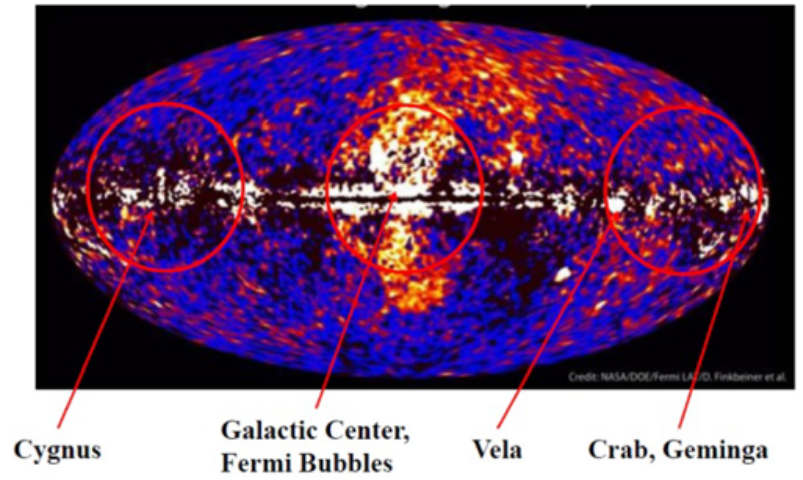

Figure 7. Galactic center, Fermi Bubbles, Crab, Cygnus, Vela, Geminga, and other regions will be observed with the GAMMA$400 \mathrm{FoV}$ of $\pm 45^{\circ}$.

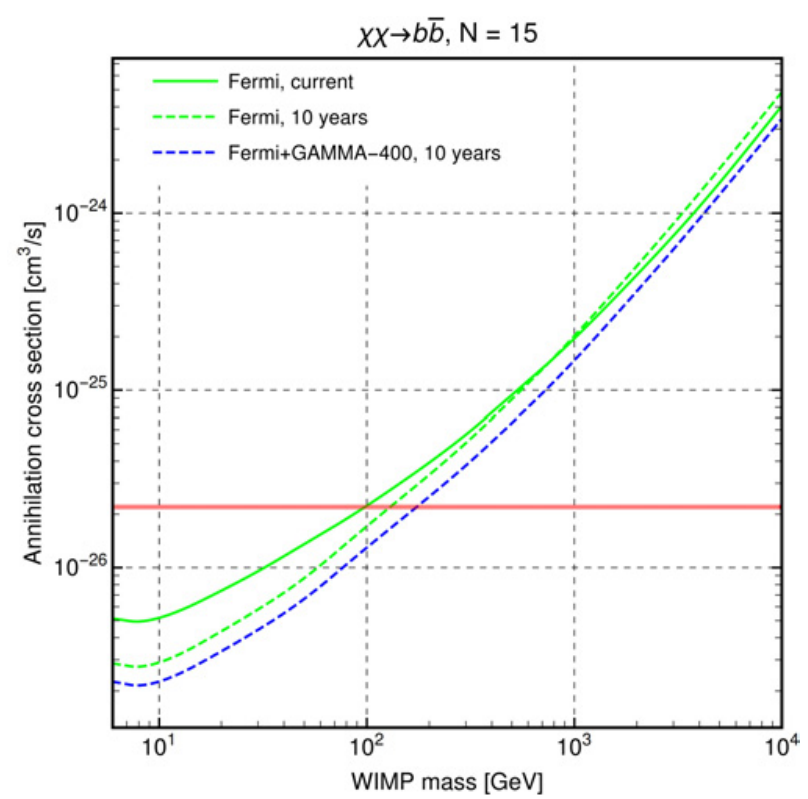

Figure 8. Current and predicted constraints for dark matter parameters according to observations of 15 known dwarf galaxies.

The main targets to search for gamma rays from dark matter are:

The Milky Way. The center of Milky Way is, apparently, the best potential source of dark matter emission possessing the largest J-factor [11]. Moreover, recently, an anomalous excess of gamma-ray emission in the $\mathrm{GeV}$ energy range was revealed near the Galactic center (the region of about one degree) [20], which can be well described by dark matter with a mass of several tens of $\mathrm{GeV}$ and annihilation cross section of about standard thermal $10^{26} \mathrm{~cm}^{3} / \mathrm{s}$. However, this observed excess can have another interpretation - the presence of a population of millisecond pulsars [21]. Therefore, the new GAMMA400 observational data can help solve this problem.

Milky Way satellites have been considered for a long time as the strongest sources of constraints for dark matter, because they have sufficiently large J-factors and at the same time have considerably less gamma-ray background in comparison with the Galactic center. Current constraints for the dark matter parameters obtained by Fermi-LAT are shown by solid lines in Fig. 8 (for the typical quarkantiquark channel [22]). The prediction for constraints for a 10-year operation of Fermi-LAT is also shown in Fig. 8. 
At present, $\sim 15$ dwarf galaxies are reliably investigated for constructing constraints. GAMMA-400 will able to specify the constrain area.

Other objects. Other potentially interesting objects are other galaxies and their clusters, where dark matter may be present and can emit gamma rays. GAMMA-400 with the highest energy resolution of $1 \%$ will have a unique sensitivity for detecting dark matter.

This work was supported by the the Space Council of Russian Academy of Sciences and the Russian Federal Space Agency.

\section{References}

[1] A. Abdo, et al., ApJ 188, 405 (2010)

[2] P. Nolan, et al., ApJS 199, 31 (2012)

[3] F. Acero, et al., ApJS 218, 23 (2015)

[4] M. Ackermann, et al., ApJS 209, 1 (2013)

[5] M. Ackermann, et al., ApJS 222, 1 (2016)

[6] R. Bühler. arXiv: 1509.00012 (2015)
[7] R.E. Ong, et al., ASR 53, 1483 (2014)

[8] D. Mazin, et al., arXiv: 1410.5073 (2014)

[9] A. Balzer, et al., arXiv : 1311.3486 (2013)

[10] CTA Consortium. Exp. Astron. 32, 193 (2011)

[11] G. Bertone. Particle dark matter - observations, models and searches (Cambridge Univ. Press, 2010)

[12] G. Bertone, et al., arXiv: 1009.5107 (2010)

[13] D. Nekrassov, et al., arXiv: 1106.2752 (2011)

[14] V. Dogiel, et al., Space Sci. Rev. 49, 215 (1988)

[15] A. Galper, et al., ASR 51, 297 (2013)

[16] A. Galper, et al., AIP Conf. Proc. 1516, 288 (2013)

[17] N. Topchiev, et al., Bull. RAS. Phys. 79, 417 (2015)

[18] N. Topchiev, et al., J. Phys.: Conf. Ser. 675 (2016) 032009

[19] S. Westerhoff, et al., ASR 53, 1492 (2014)

[20] K. Abazajian and M. Kaplinghat. Phys. Rev. D86, 083511 (2012) [arXiv: 1207.6047]

[21] R. Bartels, S. Krishnamurthy, and C. Weniger. arXiv: 1506.05104 (2015)

[22] E. Charles, et al., arXiv: 1605.02016 (2016) 